\title{
Phase structure of Gross-Neveu model with compactification in the presence of external magnetic field
}

\author{
R.N. Zhokhov ${ }^{1, a}$, P. B. Kolmakov ${ }^{3}$, V.Ch. Zhukovsky ${ }^{3}$, and K.G. Klimenko ${ }^{1,2}$ \\ ${ }^{1}$ Institute for High Energy Physics, 142281, Protvino, Moscow Region, Russia \\ 2 University "Dubna" (Protvino branch), 142281, Protvino, Moscow Region, Russia \\ ${ }^{3}$ Faculty of Physics, Moscow State University, 119991, Moscow, Russia
}

\begin{abstract}
.
In the paper there have been studied Gross-Neveu model in (2+1)-dimensional space-time with one compactified dimension in presence of external magnetic field at finite temperature. Magnetic field is directed along the uncompactified dimension that is along the axis of the cylinder on which the system lives. Chiral symmetry breaking and corresponding phase structure of the model is investigated in the leading order of $\frac{1}{N}$ expansion.
\end{abstract}

\section{Introduction}

Gross-Neveu model in the background of curved space-time and in space-time of nontrivial topology was considered in a range of papers $([3-5,9,10])$. One simple example of such spaces with nontrivial topology is $R^{d} \times S^{1} \times \cdots \times S^{1}$ space-time. Such space-times are used in models with compactified extra dimensions.

By studying models in the simplest space-time with nontrivial topology you hope to make generalization and predict properties of the models in spaces with more complicated structure .

Let us consider a very simple example, this is very important for the considered model. Let us show that in case of space-time $M^{d} \times S^{1}$ constant vector potential along compactified dimension can not be gauged away. Assume that

$$
A_{\mu}=0 \text { for } \mu=1, \ldots, d, A_{d+1}=\text { const. }
$$

Gauge transformations have the form :

$$
\begin{gathered}
\psi \rightarrow e^{i e \alpha} \psi, \\
A_{\mu} \rightarrow A_{\mu}-\partial_{\mu} \alpha .
\end{gathered}
$$

Due to the fact that boundary condition is imposed on $\psi \psi\left(x_{1}, \ldots, x_{d}, x_{d+1}+L\right)=\psi\left(x_{1}, \ldots, x_{d}, x_{d+1}\right)$, $\alpha$ satisfies the following condition :

$$
\alpha\left(x_{1}, \ldots, x_{d}, x_{d+1}+L\right)=\alpha\left(x_{1}, \ldots, x_{d}, x_{d+1}\right)+2 \pi e n,
$$

\footnotetext{
ae-mail: rzn95@mail.ru
} 
where $L$ - length of compactified dimension.

This condition is satisfied if $\alpha(x)=2 \pi n \frac{x}{L}$.

Transformation for $A_{d+1}$ has the form

$$
A_{d+1} \rightarrow A_{d+1}-\frac{2 \pi e n}{L}
$$

Hence in general $A_{d+1}$ can not be gauged away.

In this paper we consider Gross-Neveu model in $R^{2} \times S^{1}$ space-time in presence of external magnetic field directed along uncompactified dimension at finite temperature and chemical potential.

\section{Model and its thermodynamic potential}

Consider Gross-Neveu model in $R^{2} \times S^{1}$ space time (one spatial dimension is compactified), it means that our system lives on the cylinder (there are two spatial dimensions, one uncompactified and one compactified).

Let us think that the cylinder is embeded in (3+1)-dimensional Minkowski space-time, in which there is homogeneous magnetic field directed along the axis of the cylinder.

On the surface of the cylinder vector potential has only one constant component along compactified dimension. As was shown in the introduction it can not be gauged away, that is there exists non zero magnetic flux through the cylinder (along the axis of the cylinder). Moreover we assume that magnetic field interacts with the spin of the fermions, which is introduced as an additional index of the spinor field $\psi$. Note, that the spin introduced in such a way does not have any relation to Lorentz group.

Lagrangian that describes this model has the following form:

$$
\mathcal{L}=\bar{\psi}^{s} i \gamma^{\mu} D_{\mu} \psi^{s}+\frac{G}{2 N}\left(\bar{\psi}^{s} \psi^{s}\right)^{2}+\mu \bar{\psi}^{s} \gamma^{0} \psi^{s}+\frac{\sigma_{s}}{2} g \mu_{B} B \bar{\psi}^{s} \gamma^{0} \psi^{s},
$$

$\gamma$-matrices have the following form:

$$
\gamma^{0}=\sigma^{3}, \gamma^{1}=i \sigma^{1}, \gamma^{2}=i \sigma^{2}
$$

In this paper we will work in irreducible representation. In such models one often uses reducible representation in which you can define $\gamma_{5}$ matrix. Generalization to that case is obvious. Flavour index of $\psi$ is implied that runs from 1 to $N$.

Spin index s has two components 1 and 2.

Interaction of the magnetic field $B$ with the spin is introduced by the last term in the Lagrangian where $\sigma_{1,2}= \pm 1, \mu_{B}$ - Bohr magneton and $B$ - magnetic field.

$\mu$ - chemical potential, $G$ - coupling constant, covariant derivative $D_{\mu}: D_{\mu}=\partial_{\mu}-i e A_{\mu}$.

Vector potential $A_{\mu}$ has the following components: $A_{0}=0, A_{1}=0, A_{2}=$ const. Since one dimension is compactified we have to impose a boundary condition. For $\psi$ the boundary condition:

$$
\psi\left(x^{0}, x^{1}, x^{2}+L\right)=e^{2 \pi i \alpha} \psi\left(x^{0}, x^{1}, x^{2}\right)
$$

The model has $U(N)$ flavour symmetry.

Moreover the Lagrangian is invariant with respect to discrete chiral transformation which has the form:

$$
\begin{aligned}
& \psi_{L}^{\prime}\left(x_{0}, x_{1}, x_{2}\right)= \pm \psi_{L}\left(x_{0}, x_{1},-x_{2}\right), \bar{\psi}_{L}^{\prime}\left(x_{0}, x_{1}, x_{2}\right)= \pm \bar{\psi}_{L}\left(x_{0}, x_{1},-x_{2}\right), \\
& \bar{\psi}_{R}^{\prime}\left(x_{0}, x_{1}, x_{2}\right)=\mp \bar{\psi}_{R}\left(x_{0}, x_{1}-, x_{2}\right), \bar{\psi}_{R}^{\prime}\left(x_{0}, x_{1}, x_{2}\right)=\mp \bar{\psi}_{R}\left(x_{0}, x_{1},-x_{2}\right),
\end{aligned}
$$


where $\psi_{R, L}(x)=\frac{1 \pm i \gamma^{2}}{2} \psi(x), \bar{\psi}_{R, L}(x)=\bar{\psi}(x) \frac{1 \mp i \gamma^{2}}{2}$.

We can work in reducible representation and define chiral symmetry using $\gamma_{5}$ matrix but as we pointed out before this changes nothing only there will be additional degrees of freedom and by slightly changing the definition of the parameter $g$ we have doubled thermodynamic potential. It is obvious that it's imposable to define chiral symmetry using $\gamma_{5}$ matrix in irreducible representation for there is no $\gamma_{5}$ matrix. One can see that the term with the chemical potential has the same form as the term describing interaction of magnetic field with the spin thus one can combine them and write the Lagrangian in the following form:

$$
\mathcal{L}=\bar{\psi}^{s} i \gamma^{0} \partial_{0} \psi^{s}+\bar{\psi}^{s} i \gamma^{1} \partial_{1} \psi^{s}+\bar{\psi}^{s} i \gamma^{2}\left(\partial_{2}-i e A_{2}\right) \psi^{s}+\frac{G}{2 N}\left(\bar{\psi}^{s} \psi^{s}\right)^{2}+\mu_{s} \bar{\psi}^{s} \gamma^{0} \psi^{s},
$$

where we introduced two chemical potentials corresponding to particles with spin projection plus one half and minus one half $\mu_{\uparrow \downarrow}=\mu \pm \delta \mu, \delta \mu=\frac{1}{2} g \mu_{B} B$.

Let us introduce auxiliary scalar field $\sigma(x)$

$$
\mathcal{L}=\bar{\psi}^{s} i \gamma^{0} \partial_{0} \psi^{s}+\bar{\psi}^{s} i \gamma^{1} \partial_{1} \psi^{s}+\bar{\psi}^{s} i \gamma^{2}\left(\partial_{2}-i e A_{2}\right) \psi^{s}-\bar{\psi}^{s} \sigma \psi^{s}-\frac{N}{2 G} \sigma^{2}+\mu_{s} \bar{\psi}^{s} \gamma^{0} \psi^{s}
$$

Lagrangian (9) is equivalent to the Lagrangian (8), one can make sure of this using an equation of motion for the scalar field $\sigma$, which has the following form

$$
\sigma=-\frac{N}{G} \bar{\psi}^{s} \psi^{s}
$$

Let us take the functional integral over the fermion field and obtain the expression for the effective action. As a result we obtain for effective action in the leading order of in $\frac{1}{N}$-expansion.

$$
\begin{gathered}
\mathcal{S}_{\mathrm{eff}}=-i \ln \int D[\bar{\psi}(x)] D[\psi(x)] e^{i \int d^{3} x\left(\bar{\psi}\left(i \gamma^{0}\left(\partial_{0}+\mu_{s}\right)+i \gamma^{1} \partial_{1}+i \gamma^{2}\left(\partial_{2}-i e A_{2}\right)-\sigma\right) \psi-\frac{N}{2 G} \sigma^{2}\right)}= \\
=-\int d^{3} x \frac{N}{2 G} \sigma^{2}-i \ln \operatorname{det}\left(i \gamma^{0}\left(\partial_{0}+\mu_{s}\right)+i \gamma^{1} \partial_{1}+i \gamma^{2}\left(\partial_{2}-i e A_{2}\right)-\sigma\right)
\end{gathered}
$$

Let us introduce thermodynamic potential that is defined by $V_{\text {eff }}=-\frac{\mathcal{S}_{\text {eff }}}{\int d^{3} x}$.

Calculating determinant over spinor indices and using the formula $\ln d e t=\operatorname{tr} \ln$, we obtain

$$
V_{\mathrm{eff}}=\frac{N}{2 G} \sigma^{2}+i \frac{N}{L} \sum_{s=1,2} \sum_{n=-\infty}^{\infty} \int \frac{d^{2} p}{(2 \pi)^{2}} \ln \left(\left(p_{0}+\mu_{s}\right)^{2}-p_{1}^{2}-\left(\frac{2 \pi}{L}(n+\alpha)-e A_{2}\right)^{2}-\sigma^{2}\right)
$$

It is convenient to introduce Aharonov-Bohm phase $\phi,: \phi=\frac{e L A_{2}}{2 \pi}=\frac{e \pi L^{2} B}{8 \pi}$.

In the term $\left(\frac{2 \pi}{L}(n+\alpha)-e A_{2}\right)=\frac{2 \pi}{L}\left(n+\alpha-\frac{e L A_{2}}{2 \pi}\right)=\frac{2 \pi}{L}(n+\alpha-\phi)$ we denote $\alpha-\phi$ as $\alpha$, for both the phase in the boundary condition and Aharonov-Bohm phase contribute in $\alpha$.

Performing Wick rotation $p_{0} \rightarrow-i p_{0}$, we obtain

$$
V_{\mathrm{eff}}=\frac{N}{2 G} \sigma^{2}-\frac{N}{L} \sum_{s=1,2} \sum_{n=-\infty}^{\infty} \int \frac{d^{2} p}{(2 \pi)^{2}} \ln \left(\left(p_{0}-i \mu_{s}\right)^{2}+p_{1}^{2}+\left(\frac{2 \pi}{L}\right)^{2}(n+\alpha)^{2}+\sigma^{2}\right) .
$$

In order to consider our theory at finite temperature we should make use of the following prescription:

$$
\int \frac{d p_{0}}{2 \pi} f\left(p_{0}\right) \rightarrow \frac{1}{\beta} \sum_{l=-\infty}^{\infty} f\left(p_{0 l}\right), \text { where } p_{0 l}=\frac{2 \pi}{\beta}\left(l+\frac{1}{2}\right), \frac{1}{\beta}=T .
$$


We obtain for the thermodynamic potential the following expression:

$$
V_{\mathrm{eff}}=\frac{N}{2 G} \sigma^{2}-\frac{N}{\beta L} \sum_{s} \sum_{l=-\infty}^{\infty} \sum_{n=-\infty}^{\infty} \int \frac{d p_{1}}{2 \pi} \ln \left(\left(\frac{2 \pi}{\beta}\left(l+\frac{1}{2}\right)-i \mu_{s}\right)^{2}+\left(\frac{2 \pi}{L}\right)^{2}(n+\alpha)^{2}+p_{1}^{2}+\sigma^{2}\right) .
$$

\section{Calculation of the thermodynamic potential}

From now on we assume for simplicity that $\mu=0$, for in this case $\delta \mu$ plays the same role as $\mu$. If we want to obtain expression for both nonzero $\mu$ and $\delta \mu$, we need to divide the contribution by chemical potential placing before the sum $\frac{1}{2}$, then in the first part instead of chemical potential we should write $\mu+\delta \mu$ and in the second part $\mu-\delta \mu$.

Also we rewrite $\ln$ in a more convenient form making use of the fact that one can omit terms that are independent of $\sigma$.

$$
V_{\mathrm{eff}}=\frac{N}{2 G} \sigma^{2}-\frac{2 N}{\beta L} \sum_{l=-\infty}^{\infty} \sum_{n=-\infty}^{\infty} \int \frac{d p_{1}}{2 \pi} \ln \left(1+\frac{\sigma^{2}}{\left(\frac{2 \pi}{\beta}\left(l+\frac{1}{2}\right)-i \delta \mu\right)^{2}+\left(\frac{2 \pi}{L}\right)^{2}(n+\alpha)^{2}+p_{1}^{2}}\right) .
$$

We can take the sum over $l$ using the following formula:

$$
\begin{gathered}
\sum_{n=-\infty}^{\infty} \ln \left(1+\frac{b^{2}}{a^{2}+(n+\alpha)^{2}}\right)= \\
=\int d \tau \ln \left(1+\frac{b^{2}}{a^{2}+\tau^{2}}\right)+\ln \frac{1-2 \cos (2 \pi \alpha) e^{-2 \pi \sqrt{a^{2}+b^{2}}}+e^{-4 \pi \sqrt{a^{2}+b^{2}}}}{1-2 \cos (2 \pi \alpha) e^{-2 \pi \sqrt{a^{2}}}+e^{-4 \pi \sqrt{a^{2}}} .} .
\end{gathered}
$$

As a result we get the following expression for the thermodynamic potential:

$$
V_{\text {eff }}=V_{0}+V_{L}+V_{\mu T}
$$

where

$$
V_{0}=\frac{N}{2 G} \sigma^{2}-2 N \int \frac{d^{3} p}{(2 \pi)^{3}} \ln \left(p^{2}+\sigma^{2}\right)
$$

- thermodynamic potential of Gross-Neveu model without any external conditions,

$$
V_{L}=-\frac{2 N}{L} \int \frac{d^{2} p}{(2 \pi)^{2}} \ln \frac{1-2 \cos (2 \pi \alpha) \mathrm{e}^{-\mathrm{L} \sqrt{p^{2}+\sigma^{2}}}+\mathrm{e}^{-2 \mathrm{~L} \sqrt{p^{2}+\sigma^{2}}}}{1-2 \cos (2 \pi \alpha) \mathrm{e}^{-\mathrm{L} \sqrt{p^{2}}}+\mathrm{e}^{-2 \mathrm{~L} \sqrt{p^{2}}}}
$$

- contribution to the thermodynamic potential due to compactification,

$$
V_{\mu T}=-\frac{2 N}{\beta L} \sum_{n=-\infty}^{\infty} \int \frac{d p_{1}}{2 \pi}\left(\ln \left(1+e^{-\beta \sqrt{p_{1}^{2}+\sigma_{n}^{2}}+\beta \delta \mu}\right)+\ln \left(1+e^{-\beta \sqrt{p_{1}^{2}+\sigma_{n}^{2}}-\beta \delta \mu}\right)\right),
$$

where $\sigma_{n}=\sqrt{\left(\frac{2 \pi}{L}\right)^{2}(n+\alpha)^{2}+\sigma^{2}}$ - contribution to the thermodynamic potential due to temperature and chemical potential.

The expression (18) in the case of nonzero $\mu$ can be rewritten in the form

$$
V_{\mu T}=-\frac{N}{2 \beta L} \sum_{n=-\infty}^{\infty} \int \frac{d p_{1}}{2 \pi}\left(\ln \left(1+e^{-\beta E_{\uparrow}^{+}}\right)+\ln \left(1+e^{-\beta E_{\downarrow}^{+}}\right)+\ln \left(1+e^{-\beta E_{\uparrow}^{-}}\right)+\ln \left(1+e^{-\beta E_{\downarrow}^{-}}\right)\right),
$$


where $E_{s}^{ \pm}=E_{p} \pm \mu_{s}$ and $E_{p}=\sqrt{\left(\frac{2 \pi}{L}\right)^{2}(n+\alpha)^{2}+p_{1}^{2}+\sigma^{2}}$.

For $V_{0}$ we have

$$
V_{0}(\sigma)=\frac{N}{3 \pi} \sigma^{3}+\frac{N}{2 g} \sigma^{2}
$$

Rewrite the expression for $V_{L}$ with use of special functions.

$$
\begin{aligned}
V_{L}= & \frac{N}{\pi L^{3}} L i_{3}\left(e^{-L \sigma+2 \pi i \alpha}\right)+\frac{N}{\pi L^{3}} L i_{3}\left(e^{-L \sigma-2 \pi i \alpha}\right)+\frac{N \sigma}{\pi L^{2}} L i_{2}\left(e^{-L \sigma+2 \pi i \alpha}\right)+ \\
& +\frac{N \sigma}{\pi L^{2}} L i_{2}\left(e^{-L \sigma-2 \pi i \alpha}\right)-\frac{N}{\pi L^{3}} L i_{3}\left(e^{2 \pi i \alpha}\right)-\frac{N}{\pi L^{3}} L i_{3}\left(e^{-2 \pi i \alpha}\right) .
\end{aligned}
$$

where $L i_{v}(z)=\sum_{n=1}^{\infty} \frac{z^{n}}{n^{\nu}}$.

Derivative of $V_{\text {eff }}$ with respect to $\sigma$ has the following form:

$$
\begin{gathered}
\frac{d V_{\mathrm{eff}}}{d \sigma}=\frac{N}{\pi} \sigma\left(\left(\sigma-\sigma_{0}\right)+\frac{1}{L} \ln \left(1-2 \cos (2 \pi \alpha) e^{-L \sigma}+e^{-2 L \sigma}\right)\right)+ \\
+\frac{2 N \sigma}{L} \sum_{n=-\infty}^{\infty} \int \frac{d p_{1}}{2 \pi} \frac{1}{1+e^{\left(\beta \sqrt{p_{1}^{2}+\sigma_{n}^{2}}-\beta \delta \mu\right)}} \frac{1}{\sqrt{p_{1}^{2}+\sigma_{n}^{2}}}+\frac{2 N \sigma}{L} \sum_{n=-\infty}^{\infty} \int \frac{d p_{1}}{2 \pi} \frac{1}{1+e^{\left(\beta \sqrt{p_{1}^{2}+\sigma_{n}^{2}}+\beta \delta \mu\right)}} \frac{1}{\sqrt{p_{1}^{2}+\sigma_{n}^{2}}} .
\end{gathered}
$$

One can see that $\sigma=0$ is a solution of stationary equation. Besides if $\sigma \rightarrow \infty$ then $V_{\text {eff }} \rightarrow \infty$, so if at a point $\sigma=0$ the thermodynamic potential has its maximum then minimum is is located at $\sigma \neq 0$. if at a point $\sigma=0$ the thermodynamic potential has its minimum then in the absence of the other minimums this minimum is the global one. If there are other minimums then one has to compare values of the thermodynamic potential at these minimums and find the global one. It turns out that in the case of zero chemical potential $\mu=0$ without of Zeeman interaction $\delta \mu=0$ there are no other minimums and in order to find minimum of the thermodynamic potential it is enough to calculate only second derivative of the thermodynamic potential at the point $\sigma=0$.

Second derivative of the thermodynamic potential at the point $\sigma=0$ is

$$
\begin{gathered}
\left.\frac{d^{2} V_{\mathrm{eff}}}{d \sigma^{2}}\right|_{\sigma=0}=\frac{N}{\pi}\left(-\sigma_{0}+\frac{1}{L} \ln (2-2 \cos (2 \pi \alpha))\right)+ \\
+\frac{2 N}{L} \sum_{n=-\infty}^{\infty} \int \frac{d p_{1}}{2 \pi} \frac{1}{1+e^{\left(\beta \sqrt{p_{1}^{2}+\left(\sigma_{n}^{0}\right)^{2}}-\beta \delta \mu\right)}} \frac{1}{\sqrt{p_{1}^{2}+\left(\sigma_{n}^{0}\right)^{2}}}+\frac{2 N}{L} \sum_{n=-\infty}^{\infty} \int \frac{d p_{1}}{2 \pi} \frac{1}{1+e^{\left(\beta \sqrt{p_{1}^{2}+\left(\sigma_{n}^{0}\right)^{2}}+\beta \delta \mu\right)}} \frac{1}{\sqrt{p_{1}^{2}+\left(\sigma_{n}^{0}\right)^{2}}},
\end{gathered}
$$

where $\sigma_{n}^{0}=\left.\sigma_{n}\right|_{\sigma=0}$.

In the case if $\alpha$ tends to integer, $\alpha=n+\varepsilon$ when $\varepsilon \rightarrow 0$, the first term has the following asymptotic $\frac{N}{\pi L} \ln \left(\varepsilon^{2}\right)$.

Studying the behavior of other terms one can find that divergent part of second derivative of second derivative of the thermodynamic potential at the point $\sigma=0$ equals 0 :

$$
\left.\frac{d^{2} V_{\text {eff }}}{d \sigma^{2}}\right|_{\sigma=0} ^{d i v}=\frac{N}{\pi L} \ln \left(\varepsilon^{2}\right)-\frac{N}{\pi L} \ln \left(\varepsilon^{2}\right)=0 .
$$


Let us consider the case $T=0$, i.e. $\beta \rightarrow \infty$

Second derivative of the thermodynamic potential at the point $\sigma=0$ in this case has the form:

$$
\left.\frac{d^{2} V_{\mathrm{eff}}}{d \sigma^{2}}\right|_{\sigma=0}=\frac{N}{\pi}\left(-\sigma_{0}+\frac{1}{L} \ln (2-2 \cos (2 \pi \alpha))\right)+\frac{2 N}{\pi L} \sum_{n=-\infty}^{\infty} \theta\left(\mu_{n}-1\right) \ln \left(\mu_{n}+\sqrt{\mu_{n}^{2}-1}\right) \text {, }
$$

where $\mu_{n}=\frac{\delta \mu}{\frac{2 \pi}{L}|n+\alpha|}$

Let us consider the case without Zeeman interaction at zero temperature $T=0, \delta \mu=0$. In this case

$$
\frac{d V_{\mathrm{eff}}}{d \sigma}=\frac{N}{\pi} \sigma\left(\sigma-\sigma_{0}+\frac{1}{L} \ln \left(1-2 \cos (2 \pi \alpha) e^{-L \sigma}+e^{-2 L \sigma}\right)\right)=0
$$

In this case one can find an explicit expression for $\sigma$ :

$$
\sigma=\frac{1}{L} \operatorname{arcch}\left(\frac{e^{L \sigma_{0}}+2 \cos (2 \pi \alpha)}{2}\right)
$$

This expression has been obtained in the following papers [3],[4],[5] .

In the case $B=0$ if $\frac{1}{6}<\alpha<\frac{5}{6}$ there exist $L<L_{c}$, when the symmetry of the model is restored, where $L_{c}$ - critical radius of compactification:

$$
L_{c}=\frac{1}{\sigma_{0}} \ln (2-2 \cos (2 \pi \alpha))
$$

In he case of other $\alpha$ symmetry is broken for all L. It means that if $\alpha=0$ the symmetry is broken for all $\mathrm{L}$, in case of $\alpha=\frac{1}{2}: L_{c}=\frac{2 \ln 2}{\sigma_{0}}$

Let us rewrite the expression for integral $\int \frac{d p_{1}}{2 \pi} \ln \left(1+\mathrm{e}^{-\beta \sqrt{p_{1}^{2}+\sigma_{n}} \pm \beta \delta \mu}\right)$, that stand in the expression of thermodynamic potential using special functions. If $\sigma_{n}>\delta \mu$.

Make use of the formula that can be found in ([7]),

$$
\int_{a}^{\infty} x^{n+1}\left(x^{2}-a^{2}\right)^{\beta-1} e^{-p x} d x=(-1)^{n} \frac{(2 a)^{\beta+\frac{1}{2}}}{2 \sqrt{\pi}} \Gamma(\beta) \frac{\partial^{n}}{\partial p^{n}}\left[p^{\frac{1}{2}-\beta} K_{\beta+\frac{1}{2}}(a p)\right] .
$$

We obtain that

$$
\int_{-\infty}^{\infty} \frac{d p_{1}}{2 \pi} \ln \left(1+e^{-\beta \sqrt{p_{1}^{2}+\sigma_{n}^{2}} \pm \beta \delta \mu}\right)=\frac{1}{\pi} \sum_{m=1}^{\infty}(-1)^{m-1} \frac{e^{ \pm \beta \delta \mu m}}{m} \sigma_{n} K_{1}\left(\beta \sigma_{n} m\right) .
$$

As a result we get the following expression for $V_{\mu T}$

$$
\begin{gathered}
V_{\mu T}=-\frac{2 N}{\pi \beta L} \sum_{\sigma_{n}<\delta \mu} \int \frac{d p_{1}}{2 \pi} \ln \left(1+\mathrm{e}^{-\beta \sqrt{p_{1}^{2}+\sigma_{n}^{2}}+\beta \delta \mu}\right)+ \\
+\frac{2 N}{\pi \beta L} \sum_{\sigma_{n}>\delta \mu} \sum_{m=1}^{\infty}(-1)^{m} \frac{\sigma_{n}}{m} K_{1}\left(\beta \sigma_{n} m\right) \mathrm{e}^{\beta \delta \mu \mathrm{m}}+\frac{2 \mathrm{~N}}{\pi \beta \mathrm{L}} \sum_{\mathrm{n}=-\infty}^{\infty} \sum_{\mathrm{m}=1}^{\infty}(-1)^{\mathrm{m}} \frac{\sigma_{\mathrm{n}}}{\mathrm{m}} \mathrm{K}_{1}\left(\beta \sigma_{\mathrm{n}} \mathrm{m}\right) \mathrm{e}^{-\beta \delta \mu \mathrm{m}} .
\end{gathered}
$$




\section{Effective potential for which $\left.\frac{d^{2} V_{\text {eff }}}{d \sigma^{2}}\right|_{\sigma=0}$ is explicitly finite for all values of $\alpha$}

In this section we will get the expression for thermodynamic potential that has explicitly finite form of its second derivative at the point $\sigma=0$ for all values of $\alpha$.

Let us go back to the expression (15). Now take the sum over $n$ first. Making use of the formula (17), we get

$$
\begin{gathered}
V_{\mathrm{eff}}=V_{0}+V_{\beta \mu 0}+V_{L \beta \mu}, \\
V_{\beta \mu 0}=-\frac{2 N}{\beta} \int \frac{d^{2} p}{(2 \pi)^{2}} \ln \left(\frac{1+2 \operatorname{ch}(\beta \delta \mu)) e^{-\beta \sqrt{p^{2}+\sigma^{2}}}+e^{-2 \beta \sqrt{p^{2}+\sigma^{2}}}}{1+2 \operatorname{ch}(\beta \delta \mu)) e^{-\beta \sqrt{p^{2}}}+e^{-2 \beta \sqrt{p^{2}}}}\right), \\
V_{L \beta \mu}=-\frac{2 N}{\beta L} \sum_{n=-\infty}^{\infty} \int_{-\infty}^{\infty} \frac{d p_{1}}{2 \pi} \ln \left(1-2 \cos (2 \pi \alpha) e^{-L \sqrt{p_{1}^{2}+\sigma_{l}^{2}}}+\mathrm{e}^{-2 \mathrm{~L} \sqrt{p_{1}^{2}+\sigma_{l}^{2}}}\right),
\end{gathered}
$$

where $\sigma_{l}=\sqrt{\left(\frac{2 \pi}{\beta}\left(l+\frac{1}{2}\right)-i \delta \mu\right)^{2}+\sigma^{2}}$.

After not complicated calculation the expression for thermodynamic potential can be brought to the form

$$
\begin{gathered}
V_{\text {eff }}(\sigma)=\frac{N}{\pi}\left(\frac{\sigma^{3}}{3}-\frac{\sigma^{2} \sigma_{0}}{2}\right)+\sum_{ \pm} \frac{N}{\pi \beta^{3}} L i_{3}\left(-e^{-\beta \sigma \pm \beta \delta \mu}\right)+\sum_{ \pm} \frac{N \sigma}{\pi \beta^{2}} L i_{2}\left(-e^{-\beta \sigma \pm \beta \delta \mu}\right)+ \\
+\frac{4 N}{\beta L} \sum_{n=1}^{\infty} \sum_{l=-\infty}^{\infty} \frac{\sigma_{l}}{n} K_{1}\left(L \sigma_{l} n\right) \cos (2 \pi \alpha n) .
\end{gathered}
$$

The second and the third terms in the thermodynamic potential can be written in that form only if $\sigma>\delta \mu$.

Derivative of the thermodynamic potential is

$$
\begin{gathered}
\frac{d V_{\mathrm{eff}}}{d \sigma}=\frac{N}{\pi} \sigma\left(\left(\sigma-\sigma_{0}\right)+\frac{1}{\beta} \ln \left(1+2 \cosh (\beta \delta \mu) e^{-\beta \sigma}+e^{-2 \beta \sigma}\right)\right)- \\
-\frac{4 N \sigma}{\beta} \sum_{l=-\infty}^{\infty} \sum_{n=1}^{\infty} K_{0}\left(L \sigma_{l} n\right) \cos (2 \pi \alpha n) .
\end{gathered}
$$

Second derivative of the thermodynamic potential at the point $\sigma=0$ is

$$
\begin{gathered}
\left.\frac{d^{2} V_{\mathrm{eff}}}{d \sigma^{2}}\right|_{\sigma=0}=\frac{N}{\pi}\left(-\sigma_{0}+\frac{1}{\beta} \ln (2+2 \cosh (\beta \delta \mu))\right)- \\
-\frac{4 N}{\beta} \sum_{l=0}^{\infty} \sum_{n=1}^{\infty}\left(K_{0}\left(L \sigma_{l}^{+0} n\right)+K_{0}\left(L \sigma_{l}^{-0} n\right)\right) \cos (2 \pi \alpha n),
\end{gathered}
$$

where

$$
\sigma_{l}^{ \pm}=\sqrt{\left(\frac{2 \pi}{\beta}\left(l+\frac{1}{2}\right) \pm i \delta \mu\right)^{2}+\sigma^{2}}, \quad \sigma_{l}^{ \pm 0}=\left.\sigma_{l}^{ \pm}\right|_{\sigma=0} .
$$

Consider the limit $L \rightarrow \infty$ (this case has been considered in [6]). One can see that in the expression for first derivative of the thermodynamic potential (34) the last term tends to zero as $O\left(\frac{1}{L} e^{-L}\right)$. As a result we get

$$
\frac{d V_{\mathrm{eff}}}{d \sigma}=\frac{N}{\pi} \sigma\left(\left(\sigma-\sigma_{0}\right)+\frac{1}{\beta} \ln \left(1+2 \operatorname{ch}(\beta \delta \mu) e^{-\beta \sigma}+e^{-2 \beta \sigma}\right)\right)
$$


One can easily solve this for $\sigma$

$$
\sigma=\frac{1}{\beta} \operatorname{arcch}\left(\frac{e^{\beta \sigma_{0}}-2 \operatorname{ch}(\beta \delta \mu)}{2}\right) .
$$

In the case $\delta \mu<1\left(\delta \mu\right.$ is made dimensionless by $\sigma_{0}$, i.e. $\left.\delta \mu<\sigma_{0}\right)$ there exists $\beta_{c}$, such that if $\beta<\beta_{c}$ the symmetry is restored.

$\beta_{c}$ can be found from the following equation:

$$
e^{\beta \sigma_{0}}=2+e^{\beta \delta \mu}+e^{-\beta \delta \mu} .
$$

One can see that with increasing value of $\delta \mu$ the value of $\beta_{c}$ is increasing and tends to infinity if $\delta \mu \rightarrow 1$. Starting from $\delta \mu=1$ critical temperature $\beta_{c}$ does not exist, that is the symmetry is always restored for any values of $\beta$.

\section{Numeric investigation and phase portrait}

One can investigate the phase structure of the model and draw a full phase portrait of it only by numerical analysis of thermodynamic potential that is finding its global minimum point.

Let us consider the case of zero chemical potential without Zeeman interaction. In that case external magnetic field influence the system only via Aharonov-Bohm phase.

First consider the case for periodic boundary condition $\alpha=0$.

If $B=0$ (Fig. 1) one can see that by decreasing $\beta$ (that is increasing the temperature $\mathrm{T}$ ) the symmetry is restored. By increasing length of compactified dimension $L$ the curve tends to a constant value $\beta=\beta_{c}, \beta_{c}=2 \ln 2$ and this value is reached already at $L$ equals 3 .

Let us assume that external magnetic field is nonzero. By increasing $B$ one can see that there appears a bump (Fig.2), that is in corresponding region $L$ the symmetry is restored for $\beta>\beta_{c}$

At rather large $B$ (approximately $220-226$ ) the bump goes to infinity, one can see this at (Fig.3)

By further increasing $B$ there appears one more bumps (Fig. 3, 4 ), etc.

One can observe that at $B=2073$ (Fig. 5) two bumps go to infinity (though there are other bumps at the figure that do not go to infinity). This means that for infinite values of $\beta$ at some values of $\mathrm{L}$ symmetry is restored, then it breaks down and then restores again by decreasing $L$.

At large values of $B$ (Fig. 6 ) there are many bumps that go to infinity. One can see that all bumps going to infinity do not go beyond values of $L>L_{c}$ as in case of $(T=0)$. It means that magnetic field virtually do not influence the curve of phase transition of the system for $L>L_{c}$.

Let us now consider the case of antiperiodic boundary condition $\alpha=\frac{1}{2}$.

In tis case at zero magnetic field phase portrait of the system is symmetric under interchange of $\beta$ and L, a it should be according to (15) if $B=0, \alpha=\frac{1}{2}$. one can see that the curve if $L \rightarrow \infty$ tends to $\beta_{c}$, and if $\beta \rightarrow \infty$ the curve tends to $L_{c}$ (Fig.8).

If the magnetic field is nonzero $B \neq 0$, then if one increase $B$ in order to restore chiral symmetry one has to decrease length of compactified dimension $L$ to lower value than in case of $B=0$ (Fig. 7, 8). For large enough values of the magnetic field and large values of $\beta\left(\beta>\beta_{c}\right)$ there appears regions of $\mathrm{L}$, in which first the symmetry is restored, then by decreasing $L$ it breaks down and then is restored again, as it were in the case of periodic boundary condition. Values of L, at which the symmetry is restored once for all, decrease by increasing values of magnetic field, and number of regions in which the symmetry alternatively restores and breaks down becomes larger (Fig. 9, 10).

Comparing the phase portrait for zero magnetic field (Fig. 7) and for nonzero one (Fig.. 9, 10), one can see that magnetic field could break the symmetry in the region $L<L_{c}$. 
At Fig. 1-6 curve of phase transition between phase $\sigma=0$ (left) and $\sigma \neq 0$ (right) is depicted for periodic boundary condition $(\alpha=0)$ without taking into account Zeeman interaction $(\delta \mu=0)$ for different values of magnetic field $B$.

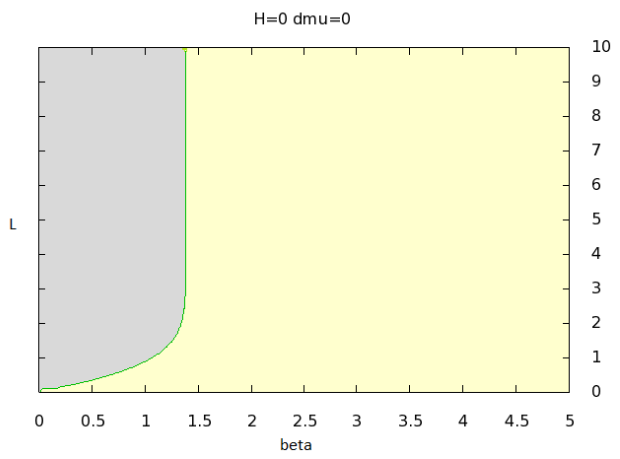

Figure 1. $\alpha=0, B=0$.

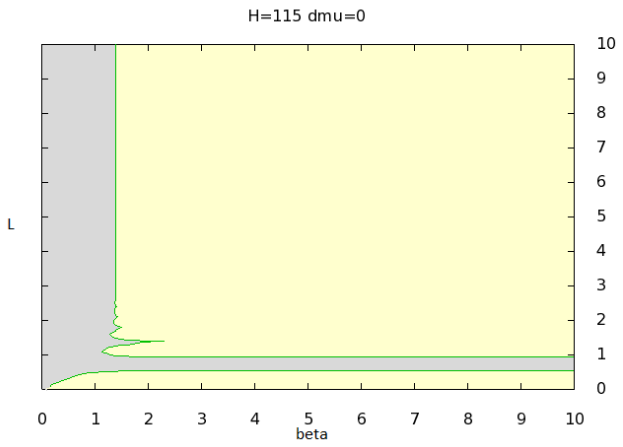

Figure 3. $\alpha=0, B=722$.

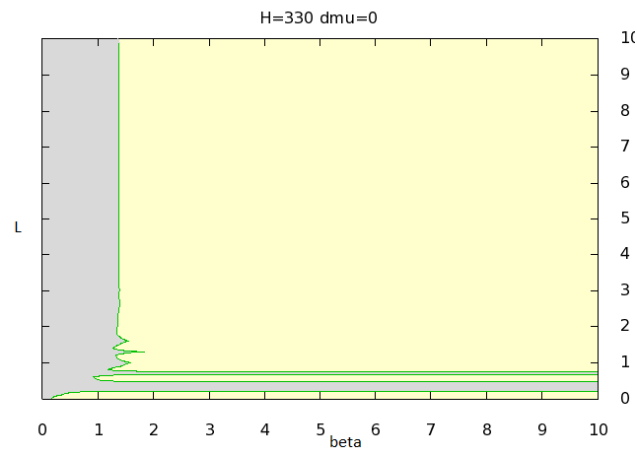

Figure 5. $\alpha=0, B=2073$.

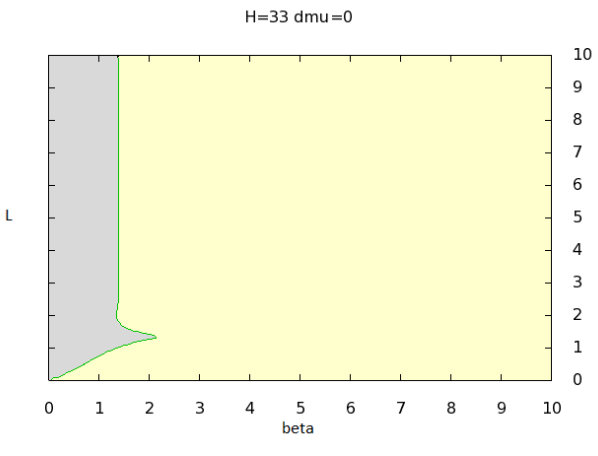

Figure 2. $\alpha=0, B=207$.

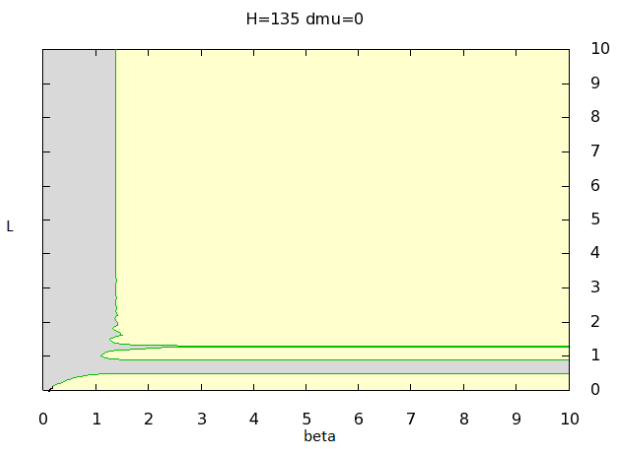

Figure 4. $\alpha=0, B=848$.

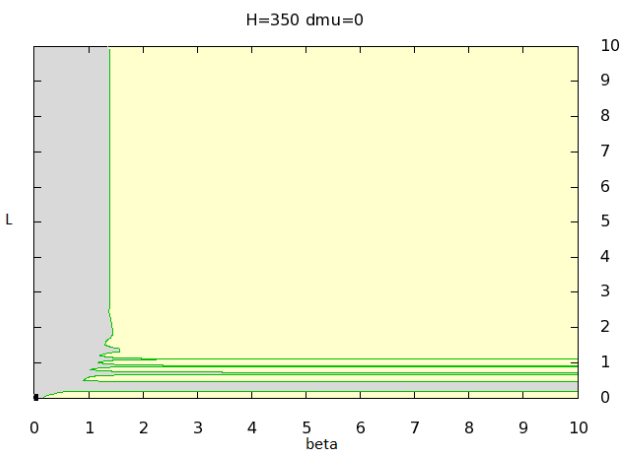

Figure 6. $\alpha=0, B=2198$. 
At Fig. 7-10 curve of phase transition between phase $\sigma=0$ (left) and $\sigma \neq 0$ (right) is depicted for antiperiodic boundary condition $\left(\alpha=\frac{1}{2}\right)$ without taking into account Zeeman interaction $(\delta \mu=0)$ for different values of magnetic field $B$.

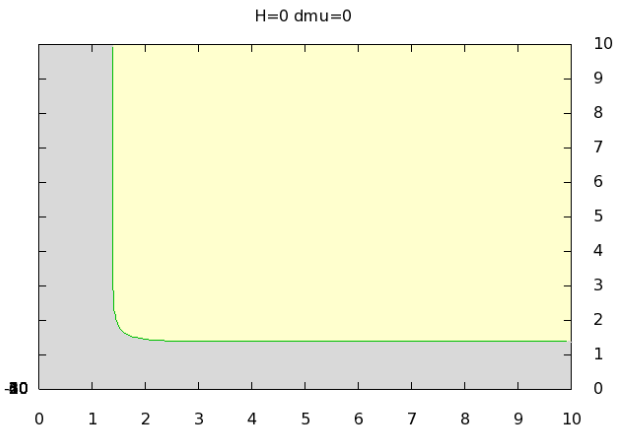

Figure 7. $\alpha=\frac{1}{2}, B=0$.

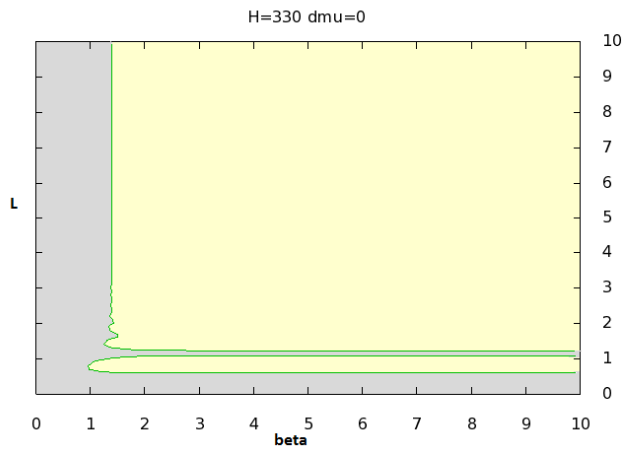

Figure 9. $\alpha=\frac{1}{2}, B=2072$.

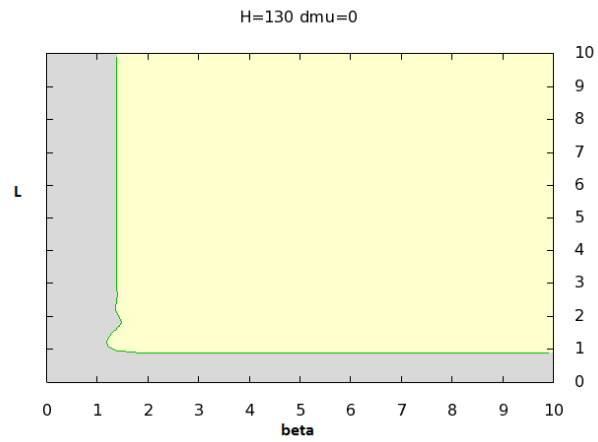

Figure 8. $\alpha=\frac{1}{2}, B=816$.

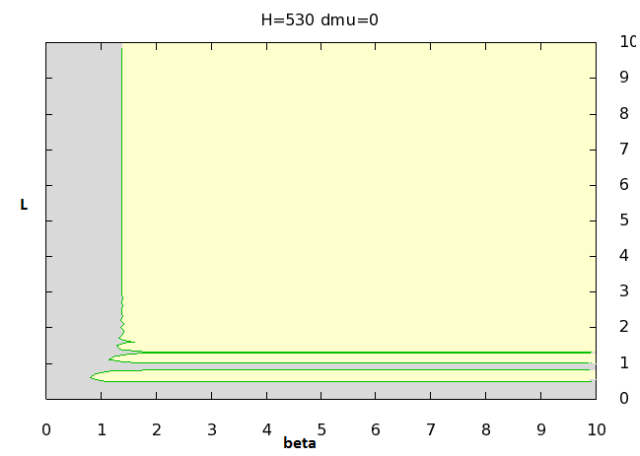

Figure 10. $\alpha=\frac{1}{2}, B=3328$.

\section{Calculation of using method of $\zeta$-regularization.}

In this section we will obtain an expression for thermodynamic potential in case of $R^{1} \times S^{1} \times S^{1}$ spacetime (the second $S^{1}$ implies that the model is considered at finite temperature), for arbitrary boundary conditions on the spinor field $\psi$, that is for arbitrary $\alpha$. We consider that there is external magnetic field $B$, but we will not take into account Zeeman interaction $\delta \mu=0$, and consider case of zero chemical potential $\mu=0$. We will make use of a method called $\zeta$-regularization. This method was used in paper [10] in order to investigate the influence of compactification and nonzero temperature on chiral symmetry breaking in two dimensional Gross-Neveu model in case of $\alpha=0, \alpha=\frac{1}{2}$. Moreover in this paper there was formulated this method for $R^{d} \times S^{1} \times S^{1}$ space time with arbitrary $d$.

Here this method is generalized for arbitrary $\alpha$ and equation for the phase portrait is obtained.

First we will obtain an asymptotic of thermodynamic potential at a point $\sigma=0$ up to terms quadratic over $\sigma$, that will allow us to get second derivative of the thermodynamic potential at the point $\sigma=0$ and determine if the point $\sigma=0$ is local minimum or maximum. In case of $\delta \mu=0$ 
thermodynamic potential always has only one minimum hence having the asymptotic at point $\sigma=0$ allows us to determine if minimum of thermodynamic potential locates at point $\sigma=0$ or at some point $\sigma \neq 0$ that is if the chiral symmetry is broken or not. Then using method of $\zeta$-regularization we will obtain an expression for thermodynamic potential in two form. This expressions coincides with those obtained using cut-off regularization.

First we will obtain asymptotic of thermodynamic potential at a point $\sigma=0$. Let us go back to the expression (15). For simplicity assume that $\mu=0$

$$
V_{\mathrm{eff}}=\frac{N}{2 G} \sigma^{2}-\frac{N}{\beta L} \sum_{s} \sum_{l} \sum_{n} \int \frac{d p_{1}}{2 \pi} \ln \left(\left(\frac{2 \pi}{\beta}\left(l+\frac{1}{2}\right)-i \delta \mu\right)^{2}+\left(\frac{2 \pi}{L}\right)^{2}(n+\alpha)^{2}+p_{1}^{2}+\sigma^{2}\right) .
$$

Using the following relation $\ln a=-\left.\left(a^{-s}\right)^{\prime}\right|_{s=0}$, we get

$$
V_{\mathrm{eff}}=\frac{N}{2 G} \sigma^{2}+\left.\Omega^{\prime}(s)\right|_{s=0}
$$

where

$$
\Omega(s)=\frac{2 N}{\beta L} \sum_{l} \sum_{n} \int \frac{d p_{1}}{2 \pi}\left(\frac{\left(\frac{2 \pi}{\beta}\left(l+\frac{1}{2}\right)-i \delta \mu\right)^{2}+\left(\frac{2 \pi}{L}\right)^{2}(n+\alpha)^{2}+p_{1}^{2}+\sigma^{2}}{\mu^{2}}\right)^{-s}
$$

and $\mu$-dimensionful parameter with the dimension of mass.

Make use of the formula

$$
a^{-s}=\frac{1}{\Gamma(s)} \int_{0}^{\infty} d t t^{s-1} e^{-a t}
$$

and if $\left(\frac{\pi}{\beta}\right)^{2}+\sigma^{2}>\delta \mu^{2}$ we obtain

$$
\Omega(s)=\frac{N}{\beta L} \sum_{l=-\infty}^{\infty} \sum_{n=-\infty}^{\infty} \int_{-\infty}^{\infty} \frac{d p_{1}}{2 \pi} \frac{1}{\Gamma(s)} \int_{0}^{\infty} d t t^{s-1} e^{-\left(\left(\frac{2 \pi}{\beta}\left(l+\frac{1}{2}\right)-i \delta \mu\right)^{2}+\left(\frac{2 \pi}{L}\right)^{2}(n+\alpha)^{2}+p_{1}^{2}+\sigma^{2}\right) \frac{t}{\mu^{2}}} .
$$

Calculating the Gaussian integral $\int_{-\infty}^{\infty} \frac{d p_{1}}{2 \pi} e^{-p_{1}^{2} t}=\frac{1}{2 \sqrt{\pi t}}$, we get

$$
\Omega(s)=\frac{N}{\beta L} \mu^{2 s} \sum_{l=-\infty}^{\infty} \sum_{n=-\infty}^{\infty} \frac{1}{2 \sqrt{\pi}} \frac{1}{\Gamma(s)} \int_{0}^{\infty} d t t^{s-\frac{3}{2}} e^{-\left(\frac{2 \pi}{\beta}\right)^{2}\left(\left(l+\frac{1}{2}\right)-\frac{i \beta \delta \mu}{2 \pi}\right)^{2} t} e^{-\left(\frac{2 \pi}{L}\right)^{2}(n+\alpha)^{2} t} e^{-\sigma^{2} t} .
$$

Let us use Poisson resummation formula

$$
\sum_{n=-\infty}^{\infty} e^{-a(n+b)^{2}}=\sqrt{\frac{\pi}{a}} \sum_{n=-\infty}^{\infty} e^{-\frac{\pi^{2} n^{2}}{a}} \cos (2 \pi b n),
$$

and transform the sum of exponents into the form

$$
\sum_{n=-\infty}^{\infty} e^{-\left(\frac{2 \pi}{L}\right)^{2}(n+\alpha)^{2} t}=\frac{L}{2 \sqrt{\pi t}}\left(1+2 \sum_{n=1}^{\infty} e^{-\frac{L^{2} n^{2}}{4 t}} \cos (2 \pi \alpha n)\right)
$$

and

$$
\sum_{l=-\infty}^{\infty} e^{-\left(\frac{2 \pi}{\beta}\right)^{2}\left(l+\frac{1}{2}-\frac{i \beta \delta \mu}{2 \pi}\right)^{2} t}=\frac{\beta}{2 \sqrt{\pi t}}\left(1+2 \sum_{l=1}^{\infty} e^{-\frac{\beta^{2} l^{2}}{4 t}}(-1)^{l} \cosh (\beta \delta \mu l)\right) .
$$


As a result for $\Omega(s)$ we obtain

$$
\begin{gathered}
\Omega(s)=\frac{N}{\sqrt{\pi} \beta L} \frac{1}{\Gamma(s)} \mu^{2 s} \int_{0}^{\infty} d t t^{s-\frac{3}{2}} \times \\
\times \frac{L}{2 \sqrt{\pi t}}\left(1+2 \sum_{n=1}^{\infty} e^{-\frac{L^{2} n^{2}}{4 t}} \cos (2 \pi \alpha n)\right) \frac{\beta}{2 \sqrt{\pi t}}\left(1+2 \sum_{l=1}^{\infty} e^{-\frac{\beta^{2} l^{2}}{4 t}}(-1)^{l} \cosh (\beta \delta \mu l)\right) e^{-\sigma^{2} t} .
\end{gathered}
$$

Generalization to the case of $\delta \mu \neq 0$ is not achieved and here we assume $\delta \mu=0$ and get:

$$
\Omega(s)=\frac{N}{4 \pi^{\frac{3}{2}}} \frac{1}{\Gamma(s)} \mu^{2 s} \int_{0}^{\infty} d t t^{s-\frac{5}{2}}\left(1+2 \sum_{n=1}^{\infty} e^{-\frac{L^{2} n^{2}}{4 t}} \cos (2 \pi \alpha n)\right)\left(1+2 \sum_{l=1}^{\infty} e^{-\frac{\beta^{2} l^{2}}{4 t}}(-1)^{l}\right) e^{-\sigma^{2} t} .
$$

There is the following relation, representation of exponent

$$
e^{-A}=\frac{1}{2 \pi i} \int_{\operatorname{Re}(z)=c>0} d z \Gamma(z) A^{-z}
$$

where one has to integrate over a contour $\operatorname{Re}(z)=c>0$. From now on wee assume that integration is over contour $\operatorname{Re}(z)=c>\frac{3}{2}$.

Using this formula we could represent exponents as contour integrals and after not complicated transformations we obtain

$$
\begin{gathered}
\Omega(s)=\frac{N \sigma^{3}}{4 \pi^{\frac{3}{2}}}\left(\frac{\mu}{\sigma}\right)^{2 s} \frac{\Gamma\left(s-\frac{3}{2}\right)}{\Gamma(s)}+ \\
+\frac{N \sigma^{3}}{4 \pi^{\frac{3}{2}}} \frac{1}{\Gamma(s)}\left(\frac{\mu}{\sigma}\right)^{2 s} \frac{1}{2 \pi i} \int d z \Gamma(z) \Gamma\left(z+s-\frac{3}{2}\right) \sum_{(l, n) \neq(0,0)} \frac{(-1)^{l} \cos (2 \pi \alpha n)}{\left(\beta^{2} l^{2}+L^{2} n^{2}\right)^{z}}\left(\frac{\sigma}{2}\right)^{-2 z} .
\end{gathered}
$$

In order to get $V_{\text {eff }}$, one has to take a derivative with respect to $s$ at a point $s=0$. In both terms nonzero contribution is provided from deriving of $\Gamma$-function in denominator. We get a function

$$
\begin{gathered}
\left.\frac{d}{d s}\left(\frac{1}{\Gamma(s)}\right)\right|_{s=0}=-\left.\frac{\Gamma^{\prime}(s)}{\Gamma^{2}(s)}\right|_{s=0}=-\left.\frac{\psi(s)}{\Gamma(s)}\right|_{s=0}=1 \\
\psi(s)=\frac{\Gamma^{\prime}(s)}{\Gamma(s)} .
\end{gathered}
$$

$\psi$ has poles of 1 -st order at points $s=-1,-2,-3, \ldots$.

$\operatorname{Res}_{s=0}(\psi(s))=-1$, for $\psi(z+1)=\psi(z)+\frac{1}{z}$.

As a result the first term in (43) together with the first term in (38) give the following contribution to thermodynamic potential:

$$
\frac{N}{2 G_{r}} \sigma^{2}+\frac{N \sigma^{3}}{3 \pi}
$$

where - $G_{r}$-renormalized coupling constant, subscript $r$ we inserted after $\zeta$-regularization of expression for thermodynamic potential. In reality it remains literally the same and equals the coupling constant $G$.

Using the following notation $\frac{1}{G_{r}}=-\frac{\sigma_{0}}{\pi}$, one can see that the expression for thermodynamic potential in the case $L=\infty, \beta=\infty, \mu=0, \delta \mu=0, B=0$ coincides with (19), when cut-off regularization was used. 
Let us introduce a function $Z$

$$
Z(a, b, \alpha, s)=\sum_{(l, n) \neq(0,0)} \frac{\cos (2 \pi \alpha n)}{\left(a^{2} l^{2}+b^{2} n^{2}\right)^{s}},
$$

which in case of $\alpha=0$ is reduced to Epshtein $Z$-function

$$
Z(a, b, s)=\sum_{(n, m) \neq(0,0)} \frac{1}{\left(a^{2} n^{2}+b^{2} m^{2}\right)^{s}} .
$$

Let us write the relation which helps to bring the sum in the expression for $V_{\text {eff }}$, to $Z$-function.

$$
\begin{gathered}
\sum_{(n, m) \neq(0,0)} \frac{(-1)^{n}}{\left(a^{2} n^{2}+b^{2} m^{2}\right)^{s}}=2 Z(2 a, b, s)-Z(a, b, s), \\
\sum_{(n, m) \neq(0,0)} \frac{(-1)^{n+m}}{\left(a^{2} n^{2}+b^{2} m^{2}\right)^{s}}=Z(a, b, s)-2 Z(2 a, b, s)-2 Z(a, 2 b, s)+4 Z(2 a, 2 b, s), \\
\sum_{(l, n) \neq(0,0)} \frac{(-1)^{l} \cos (2 \pi \alpha n)}{\left(a^{2} l^{2}+b^{2} n^{2}\right)^{s}}=2 Z(2 a, b, \alpha, s)-Z(a, b, \alpha, s) .
\end{gathered}
$$

An integral in the expression (43) for $V_{\text {eff }}$ in the case of $\alpha=0$ with the help of formula (44), and in the case of $\alpha=\frac{1}{2}$ with the help of formula (45), can be brought down to the integral of the form

$$
I=\frac{N \sigma^{3}}{4 \pi^{\frac{3}{2}}} \frac{1}{2 \pi i} \int d z \Gamma(z) \Gamma\left(z-\frac{3}{2}\right) Z(\beta, L, z)\left(\frac{\sigma}{2}\right)^{-2 z} .
$$

It can be shown that a function $\Gamma(z) Z(\beta, L, z) \Gamma\left(z-\frac{3}{2}\right)$ has poles at points $z=\frac{3}{2}-n, n=0,1 \ldots$ due to $\Gamma\left(z-\frac{3}{2}\right)$ and at points $z=0, z=1$ due to $\Gamma(z) Z(\beta, L, z)$.

The pole at $z=\frac{3}{2}$ gives a result independent of $\sigma$, and can be omitted. The pole at $z=1$ gives a term proportional to $\sigma$. The pole at $z=\frac{1}{2}$ gives a term proportional to $\sigma^{2}$. Other poles gives higher orders of $\sigma$.

Using the relation $\Gamma\left(-\frac{1}{2}\right)=-2 \sqrt{\pi}$ one can get

$$
Z\left(\beta, L, \frac{1}{2}\right)=\frac{8}{\beta} \sum_{m=1}^{\infty} \sum_{n=1}^{\infty} K_{1}\left(2 \pi \frac{L}{\beta} n m\right) \cos (2 \pi \alpha m) .
$$

As a result

$$
I=-\frac{N}{2} \frac{1}{\beta L} \sigma-\frac{2 N \sigma^{2}}{\pi \beta} \sum_{m=1}^{\infty} \sum_{n=1}^{\infty} K_{1}\left(2 \pi \frac{L}{\beta} n m\right) \cos (2 \pi \alpha m)+O\left(\sigma^{3}\right) .
$$

The first term gives no contribution to thermodynamic potential (it can be seen from (44), (45)). As a result for $V_{\text {eff }}$ in the case of $\alpha=0$ we get

$$
V_{\mathrm{eff}}=\frac{N}{\pi}\left(\frac{\sigma^{3}}{3}-\frac{\sigma^{2} \sigma_{0}}{2}\right)+\frac{2 N \sigma^{2}}{\pi \beta} \sum_{m=1}^{\infty} \sum_{n=1}^{\infty}\left(K_{1}\left(2 \pi \frac{L}{\beta} n m\right)-K_{1}\left(\pi \frac{L}{\beta} n m\right)\right)+O\left(\sigma^{3}\right) .
$$


In the case of $\alpha=\frac{1}{2} V_{\mathrm{eff}}$ has the form

$$
\begin{gathered}
V_{\mathrm{eff}}=\frac{N}{\pi}\left(\frac{\sigma^{3}}{3}-\frac{\sigma^{2} \sigma_{0}}{2}\right)+\frac{2 N \sigma^{2}}{\pi \beta} \sum_{m=1}^{\infty} \sum_{n=1}^{\infty}\left(2 K_{1}\left(4 \pi \frac{L}{\beta} n m\right)-2 K_{1}\left(2 \pi \frac{L}{\beta} n m\right)+\right. \\
\left.+K_{1}\left(\pi \frac{L}{\beta} n m\right)-K_{1}\left(2 \pi \frac{L}{\beta} n m\right)\right)+O\left(\sigma^{3}\right) .
\end{gathered}
$$

For arbitrary value of $\alpha$ we get for thermodynamic potential

$$
V_{\mathrm{eff}}=\frac{N}{\pi}\left(\frac{\sigma^{3}}{3}-\frac{\sigma^{2} \sigma_{0}}{2}\right)+\frac{2 N \sigma^{2}}{\pi \beta} \sum_{m=1}^{\infty} \sum_{n=1}^{\infty}\left(K_{1}\left(2 \pi \frac{L}{\beta} n m\right)-K_{1}\left(\pi \frac{L}{\beta} n m\right)\right) \cos (2 \pi \alpha m)+O\left(\sigma^{3}\right) .
$$

Now let us obtain expressions for thermodynamic potential in case of $v \neq 0$, using method of $\zeta$-regularization. In order to do that let us go back to the expression (42)

$$
\begin{gathered}
\tilde{\Omega}(s)=\frac{N}{4 \pi^{\frac{3}{2}}} \frac{\mu^{2 s}}{\Gamma(s)} \int_{0}^{\infty} d t t^{s-\frac{5}{2}} e^{-\sigma^{2} t}+\frac{N}{2 \pi^{\frac{3}{2}}} \frac{\mu^{2 s}}{\Gamma(s)} \int_{0}^{\infty} d t t^{s-\frac{5}{2}} \sum_{n=1}^{\infty} e^{-\frac{L^{2} n^{2}}{4 t}-\sigma^{2} t} \cos (2 \pi \alpha n)+ \\
\quad+\frac{N}{\sqrt{\pi} \beta L} \frac{1}{\Gamma(s)} \mu^{2 s} \int_{0}^{\infty} d t t^{s-\frac{3}{2}} \times \\
\times \frac{L}{2 \sqrt{\pi t}}\left(1+2 \sum_{n=1}^{\infty} e^{-\frac{L^{2} n^{2}}{4 t}} \cos (2 \pi \alpha n)\right) \frac{\beta}{2 \sqrt{\pi t}} 2 \sum_{l=1}^{\infty} e^{-\frac{\beta^{2} l^{2}}{4 t}}(-1)^{l} \operatorname{ch}(\beta v l) e^{-\sigma^{2} t} .
\end{gathered}
$$

In the third term of (50) we can use Poisson resummation formula backwards we get for thermodynamic potential

$$
\begin{gathered}
V_{\mathrm{eff}}=\frac{N}{\pi}\left(\frac{\sigma^{3}}{3}-\frac{\sigma^{2} \sigma_{0}}{2}\right)+\frac{N}{2 \pi^{\frac{3}{2}}} \int_{0}^{\infty} d t t^{-\frac{5}{2}} \sum_{n=1}^{\infty} e^{-\frac{L^{2} n^{2}}{4 t}-\sigma^{2} t} \cos (2 \pi \alpha n)+ \\
+\frac{N}{\pi L} \int_{0}^{\infty} d t t^{-2} \sum_{n=-\infty}^{\infty} \sum_{l=1}^{\infty} e^{-\frac{\beta^{2} l^{2}}{4 t}-\sigma_{n}^{2} t}(-1)^{l} \operatorname{ch}(\beta v l) .
\end{gathered}
$$

Second term in the expression for $V_{\text {eff }}$ we can transform to

$$
\frac{2 N}{\pi L^{3}} \sum_{n=1}^{\infty} \frac{e^{-\sigma L n}}{n^{3}} \cos (2 \pi \alpha n)+\frac{2 N \sigma}{\pi L^{2}} \sum_{n=1}^{\infty} \frac{e^{-\sigma L n}}{n^{2}} \cos (2 \pi \alpha n) .
$$

The third term in the expression for $V_{\text {eff }}$ can be transformed to

$$
\begin{gathered}
\frac{2 N}{\pi L \beta} \sum_{\sigma_{n}>v} \sum_{l=1}^{\infty} \frac{\sigma_{n}}{l} K_{1}\left(\beta \sigma_{n} l\right)(-1)^{l} e^{\beta v l}+\frac{2 N}{\pi L \beta} \sum_{n=-\infty}^{\infty} \sum_{l=1}^{\infty} \frac{\sigma_{n}}{l} K_{1}\left(\beta \sigma_{n} l\right)(-1)^{l} e^{-\beta v l}+ \\
+\frac{N}{2 \pi L} \int_{0}^{\infty} d t t^{-2} \sum_{\sigma_{n}<v} \sum_{l=1}^{\infty} e^{-\frac{\beta^{2} l^{2}}{4 t}-\sigma_{n}^{2} t}(-1)^{l} e^{\beta v l} .
\end{gathered}
$$

One can see that for $\sigma>v$ expression for thermodynamic potential coincides with the expression (29). 
Now let us obtain an expression for thermodynamic potential in another form. Again go back to the expression (41), now apply Poisson resummation formula backwards to sum over $l$.

For thermodynamic potential we get

$$
\begin{gathered}
V_{\text {eff }}=\frac{N}{\pi}\left(\frac{\sigma^{3}}{3}-\frac{\sigma^{2} \sigma_{0}}{2}\right)+\frac{N}{2 \pi^{\frac{3}{2}}} \int_{0}^{\infty} d t t^{-\frac{5}{2}} \sum_{l=1}^{\infty} e^{-\frac{\beta^{2} l^{2}}{4 t}-\sigma^{2} t}(-1)^{l} \operatorname{ch}(\beta v l)+ \\
+\frac{N}{\pi L} \sum_{l=-\infty}^{\infty} \sum_{n=1}^{\infty} \int_{0}^{\infty} d t t^{-2} e^{-\frac{L^{2} n^{2}}{4 t}-\sigma_{l}^{2} t} \cos (2 \pi \alpha n) .
\end{gathered}
$$

For $\sigma>v$ the second term can be transformed to

$$
\frac{N}{\pi \beta^{3}}\left(L i_{3}\left(-e^{-\beta l(\sigma+v)}\right)+L i_{3}\left(-e^{-\beta l(\sigma-v)}\right)+\beta \sigma\left(L i_{2}\left(-e^{-\beta l(\sigma+v)}\right)+L i_{2}\left(-e^{-\beta l(\sigma-v)}\right)\right) .\right.
$$

The third one can be transformed to

$$
\frac{4 N \sigma}{\pi \beta L} \sum_{l=-\infty}^{\infty} \sum_{n=1}^{\infty} \frac{\sigma_{l}}{n} K_{1}\left(L \sigma_{l} n\right) \cos (2 \pi \alpha n) .
$$

One can see that this expression coincides with the expression (33).

\section{Conclusions and some numerical estimation}

There have been obtained expression for thermodynamic potential in two forms each is useful in different situations.

In case of periodic $(\alpha=0)$, if there is nonzero magnetic flux then it can restore the symmetry at zero temperature. However, the symmetry can be restored only for not very large radius $L<L_{c}$.

In the case of antiperiodic boundary condition $\left(\alpha=\frac{1}{2}\right)$ phase portrait of the system is symmetric in the plane of $(L, \beta)$, that is compactification restores the symmetry of the model as increasing of temperature ( $\beta$ is analogous to $L$ ). In some situation in this case magnetic field can break the symmetry.

For all boundary condition when magnetic fields are large there is a region of $L$, where the symmetry can be restored and broken down several times by not so large changes of $L$.

Using method of $\zeta$-regularization we obtained the expression for the thermodynamic potential and its behavior at zero, which can be used in determining if zero is local minimum or local maximum of the thermodynamic potential.

Let us do some numerical estimation.

One can see that magnetic field always stands in the expression $\frac{\Phi}{\Phi_{0}}$, where $\Phi$ - magnetic flux, $\Phi_{0}=\frac{2 \pi}{e}$,

$\Phi=L A_{2}=\frac{L^{2} B}{4 \pi}$. Magnetic flux contribute if at least $\frac{\Phi}{\Phi_{0}} \sim 10^{-2}$. Assume that $L \sim 10^{-7} \mathrm{~cm}$.

Let us estimate magnetic field that correspond to such magnetic flux.

$$
\frac{\Phi}{\Phi_{0}}=\frac{e L^{2} B}{8 \pi^{2}}=\frac{1}{8 \pi^{2}}\left(\frac{L}{\lambda_{c}}\right)^{2} \frac{B}{B_{c}},
$$

where $B_{c}=\frac{m^{2}}{e} \sim 10^{13} \mathrm{G}, \lambda_{c}=\frac{1}{m}$-Compton wavelength. Restoring $\hbar, c$, we write $\lambda_{c}=\frac{\hbar}{m c} \sim 10^{-13} \mathrm{~m}$. Instead of speed of light $c$ if we consider application to graphene we should take Fermi velocity $v_{F}=\frac{c}{300}$, and in the following estimation we should perform transformation $L \rightarrow v_{F} L$. Estimate for the field is $B \sim 10^{3} \mathrm{G}=0.1 \mathrm{~T}$. 


\section{References}

[1] D.J. Gross and A. Neveu, Phys. Rev. D 10, 3235 (1974).

[2] A.S. Vshivtsev, B.V. Magnitsky, V.C. Zhukovsky and K.G. Klimenko, Phys. Part. Nucl. 29, 523 (1998) [Fiz. Elem. Chast. Atom. Yadra 29, 1259 (1998)].

[3] D. Y. Song, Phys.Rev D v.48, n 8, 1993

[4] A.V. Gamayun and E.V. Gorbar, arXiv:hep-ph 0411157v2, 2004

[5] I. V. Krive and S. A. Naftulin, Nuclear Physics B364 (1991) 541-550, 1991

[6] H. Caldas and R.O. Ramos, Phys. Rev. B 80, 115428 (2009).

[7] Integrals and series

A.P. Prudnikov, J.A. Bruchkov, O.I. Marichev, "Nauka", 1981

[8] D. Ebert and K. G. Klimenko, Phys.Rev. D 82, 0250182010

[9] D. Ebert, K.G. Klimenko, Phys. Rev. D80, 125013 (2009).

[10] A.S.Vshivtsev, A.G.Kisunko, K.G. Klimenko, D.V. Peregudov, IHEP 96-58 